\title{
Physical assessment of toxicology at nanoscale: nano dose-metrics and toxicity factor
}

\author{
P. P. Pompa, " G. Vecchio, A. Galeone, V. Brunetti, G. Maiorano, S. Sabella and R. Cingolani \\ Received 3rd March 2011, Accepted 28th March 2011 \\ DOI: $10.1039 / \mathrm{c} 1 \mathrm{nr} 10233 \mathrm{~h}$
}

\begin{abstract}
In this work, we propose a systematic and reproducible evaluation of nanoparticles (NPs) toxicology in living systems, based on a physical assessment and quantification of the toxic effects of NPs by the experimental determination of the key parameter affecting the toxicity outcome (i.e., the number of NPs) and of the NPs "toxicity factor". Such a strategy was applied to a well determined scenario, i.e., the ingestion of citrate-capped gold NPs (AuNPs) of different sizes by the model system Drosophila melanogaster. Using these AuNPs as a reference toxicity standard, we were able to define different regions in the multiparametric space of toxicity, enabling the classification of the toxic levels of other nanomaterials, such as quantum dots and pegylated AuNPs. This approach may pave the way to a systematic classification of nanomaterials, leading to important developments in risk assessment and regulatory approval, as well as in a wide range of nanomedicine applications.
\end{abstract}

\section{Introduction}

The ever increasing use of nanoscale manufacturing technologies is leading to a dramatic augmentation of human exposure to nanoparticles over the last decade. Lifetime prolonged exposure of living systems to nanosized objects (below $100 \mathrm{~nm}$ ) occurs primarily by inhalation (connected to environmental pollution), ingestion (connected to food and water) and dermal contact (connected to contact with nanocomposites and/or artificial materials). The up-taken nanoparticles (NPs) may have different size, shape, chemical composition, and surface chemistry, resulting in different surface-to-volume ratio and chemical reactivity of the species. ${ }^{1}$ Since the immune system of the human body is not designed to detect and recognize unknown intruders such as NPs, the determination of the overall kinetic effects of the NP-cell interaction in vivo and the assessment of the actual toxicity is a complex multiparametric problem, in which the type of entrance route in the body determines the cellular system coming in contact with the NP, whereas the physical-chemical properties of the nanoparticle determine the specific biological response of the organ involved. Noteworthy, both in vitro and in vivo, the NPs interact with biological fluids and are typically covered by a protein corona, which depends on the cellular environment. $^{2-4}$ This changes the original physical-chemical properties of the particle, further complicating the problem.

Despite the issue of toxicology of nanosystems has paramount societal implications for public health in all advanced countries, to date no systematic protocol for the nanotoxicology assessment

Center for Bio-Molecular Nanotechnology, Italian Institute of Technology (IIT), Via Barsanti, 1, 73010 Arnesano, Lecce, Italy. E-mail: pierpaolo. pompa@iit.it; Fax: +39-0832-295708; Tel: +39-0832-295714 exists. Yet, the large number of studies recently reported in the literature, though clearly demonstrating a general toxicity of NPs in living systems, often lead to controversial conclusions because of the different choices of the many possible parameters characterizing the toxicology studies (cell lines, dose metrics, different sources of NPs, NPs characterization, etc.). ${ }^{1,5}$

In this work, we propose a systematic and reproducible assessment of NPs toxicology in living systems, focusing on the dose metrics of NPs, and investigating how a specific metrics (e.g., mass, surface area, number of particles) can directly influence the toxic effects observed in vivo. To this purpose, in the multiparametric space of nanotoxicology, we fixed several parameters related to the chemical-physical characteristics of the NPs (namely, composition, shape, surface chemistry, and surface charge), varying only one factor per experiment, in order to understand which NPs characteristic plays a key role in determining their toxicity. By this approach, we elucidated whether size-dependent toxic effects observed in vivo are due to the NPs dimension, to their exposed surface area, or if toxicity is mainly determined by the total number of up-taken NPs. Such a strategy was applied to a well determined scenario, i.e., the ingestion of citrate-capped gold NPs (AuNPs) of different sizes (5, 15, 40, $80 \mathrm{~nm}$ ) by the model system Drosophila melanogaster. This is representative of a class of toxicology issues connected with the ingestion of food or water, polluted or stored in metallic containers, or the use of tooth paste/tooth brushes containing metallic NPs as antibacterial agents. After defining a doseresponse curve for AuNPs ingestion in Drosophila, we characterized such specific type of NPs by a "toxicity factor", based on some specific parameters derived from the mathematical fitting of the observed toxicity in the model organism. Using these AuNPs as a toxicity standard, to thus define a universal toxicity 
scale, ranging from highly toxic to biocompatible nanomaterials. We exploit such toxicity scale to investigate and classify the toxic effect of two additional NPs: (i) a similar AuNP but with a different surface coating; (ii) a semiconductor quantum dot (QD), finding the former nearly biocompatible and the latter highly toxic.

\section{Results and discussion}

Highly controlled citrate-capped spherical AuNPs of different sizes were synthesized in aqueous phase under ambient conditions (see Methods). They were metrologically assessed by UVvis, DLS, zeta-potential, TEM, to be spherical, in four families of diameter $5 \mathrm{~nm}, 15 \mathrm{~nm}, 40 \mathrm{~nm}, 80 \mathrm{~nm}$, with size dispersion better than $6 \%$ (Fig. 1).

The ingestion uptake is induced in Drosophila melanogaster, a well established model system for toxicological ${ }^{6-11}$ and human disease $^{12-18}$ studies, with a genome that shares most gene families and pathways with mammals $(75 \%$ of all human disease genes have related sequences in D. melanogaster), ${ }^{19}$ as well as some of the same tissues and organ systems. ${ }^{20}$ Flies have several distinct experimental advantages, such as their rapid life cycle and the large numbers of individuals that can be generated, which make them ideal for genetic screens and analysis of complex multigenic disorders. $^{21}$

In order to assess the key parameters involved in NPs toxicity, we define the toxicity functional $T$, as $T[S(R), x, s c, c, c l, N]$, where $S(R)$ is the surface area of the particle, in turn given by the radius $R, x$ the chemical composition, sc the surface chemistry on the NP, $c$ its surface charge, cl the type of cell line (i.e., the tissue targeted by the NP), and $N$ the number of particles ingested by the animal. As for our experiments we used four different sizes of otherwise identical AuNPs and one single administration modality (ingestion), at first approximation, we can write $T[S(R)$, $x, s c, c, c l, N]=T[S(R), N]$.

The two parameters have to be controlled with high precision and independently in order to assess whether the surface (i.e., the size) or the number of NPs is more relevant for toxicity. In the former case, the lifespan of the tested animal system depends on the Total Exposed Surface area (TES), given by $\operatorname{TES}(R)=$ $S(R) \times N$. Since the surface-to-volume ratio of the NPs scales with $1 / R$ and the surface itself with $R^{2}$, it is very important to control the radius with very high precision, as in our experiments (radius dispersion $\Delta R \leq 6 \%$ ). In the second case, the lifespan of the tested animal system depends on the Total Number of Nanoparticles ingested (TNN). This can be estimated taking the statistically determined average daily volume of food taken by a Drosophila $(V=1.5 \pm 0.04 \mu \mathrm{L} \text { per day })^{22}$ times the concentration of NPs intentionally introduced in their food.

In our first set of experiments, we tested the dependence of the $T$ functional on the surface area (see Table in Fig. 2). Controlled Drosophila populations were fed during their lifespan with the four AuNP families $(5,15,40,80 \mathrm{~nm})$ of decreasing concentration, so to keep the up-taken TES $(R)$ constant, independent of the NPs size (independent of $R$ ). The results are shown in Fig. 2. The highly controlled metrological standard of our NPs allowed us to draw quantitative conclusions in terms of Drosophila response to the AuNPs. We found that despite the TES is constant, the lifespan of Drosophila decreases dramatically with decreasing the size of the NPs. Drosophila fed with a constant TES of relatively large AuNPs $(80 \mathrm{~nm})$ exhibited a modest decrease of the lifespan compared to the reference population (fed with the same food without NPs). Drosophila fed with the small NPs $(5 \mathrm{~nm})$ underwent a significant lifespan reduction of a factor of $c a .2$ (with a $\tau_{50}$ reduced to 48 days with respect to 82 days of the control, see inset of Fig. 2). Overall, these results indicate that the ingestion of small metallic NPs is indeed toxic, but the toxicity does not depend on the TES of the NPs. Given the same composition, shape, surface chemistry and surface charge (sub-100 nm citrate-capped Au nanospheres), and the same administration method (ingestion), the overall toxicity is instead governed by the absolute number of the NPs ingested, i.e. $T[S(R), N]=T[N]$. In fact, even though the lifespan seems to decrease with decreasing the NPs radius $(R)$, such a decrease is actually due to the increasing concentration (i.e., number of NPs) of the smaller NPs (the concentration of the $5 \mathrm{~nm}$ NPs is more than two orders of magnitude higher than that of the $80 \mathrm{~nm}$ particles, see table in Fig. 2).

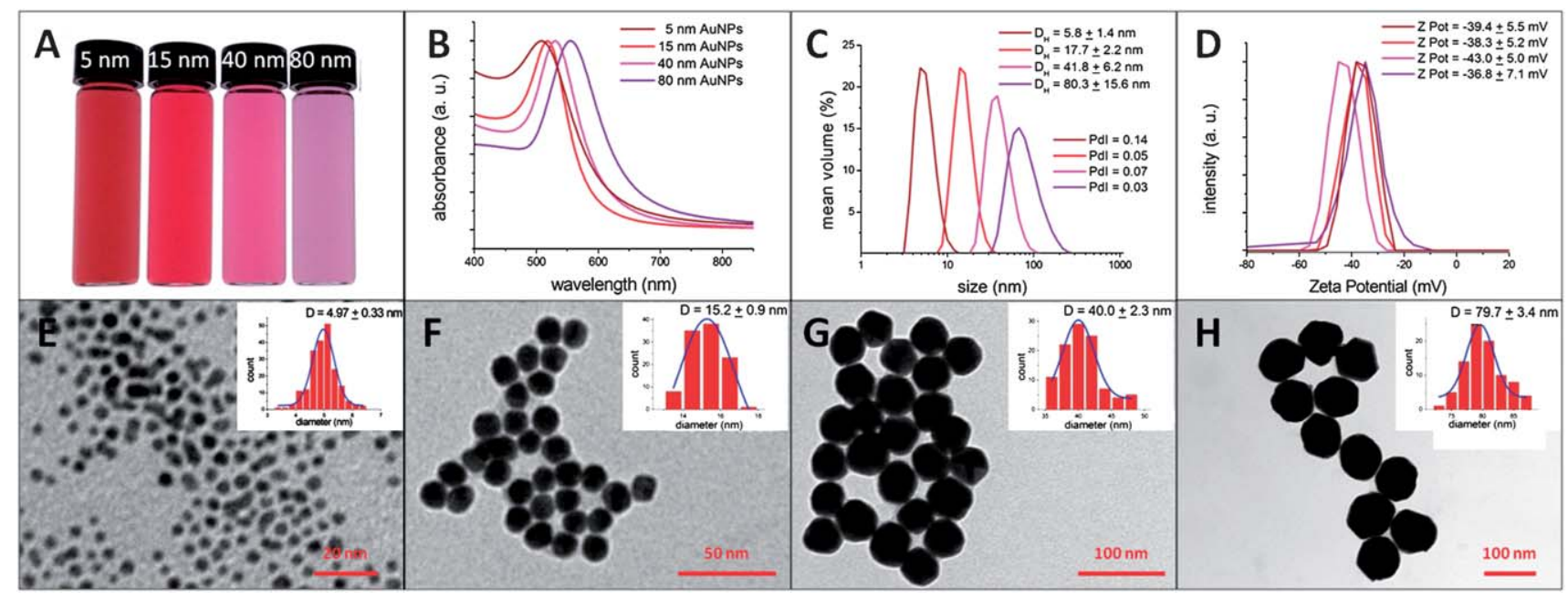

Fig. 1 (A) Photograph of a vial of 5, 15, 40, and $80 \mathrm{~nm}$ citrate-capped AuNPs freshly prepared; (B) SPR absorption spectra; (C) DLS analyses and (D) $Z$-potential analyses; (E-H) representative TEM image and size distribution analyses obtained from more than 100 NPs in random fields. 

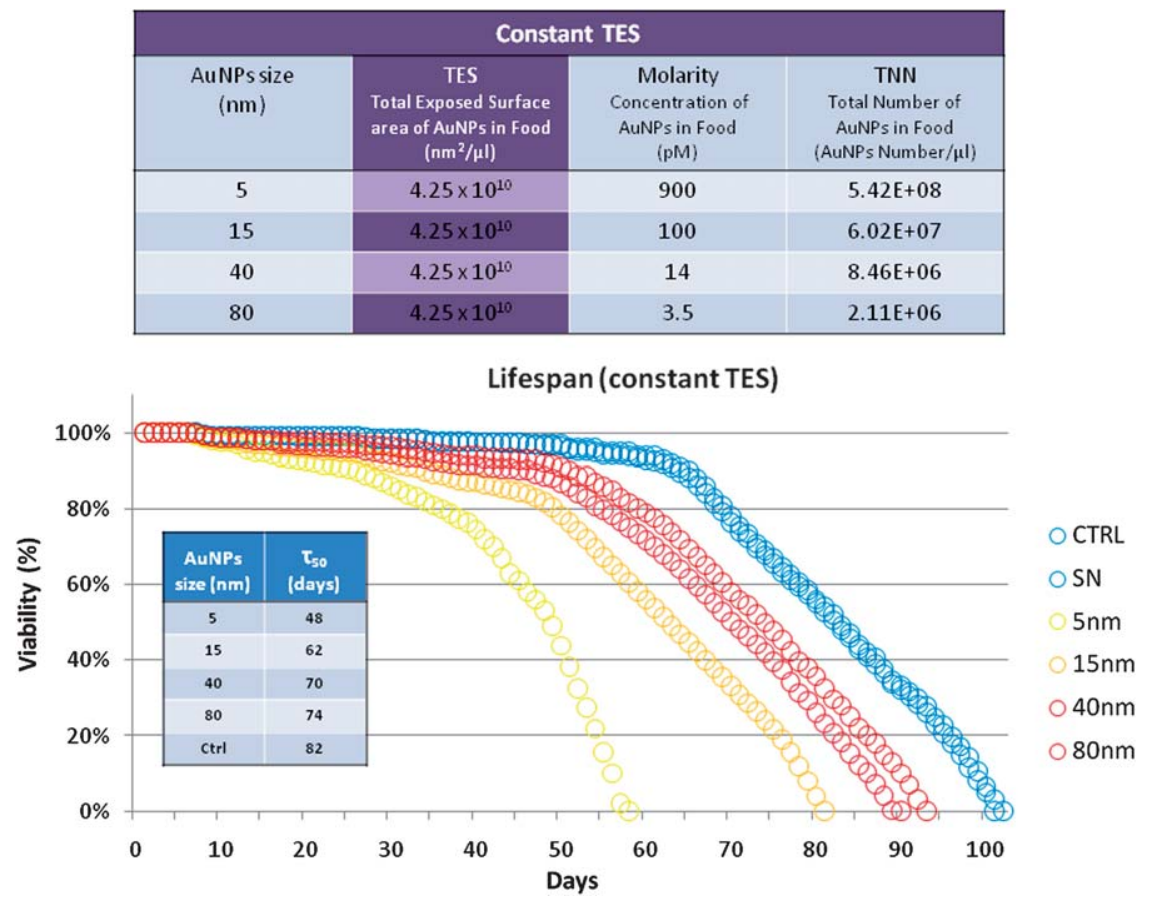

Fig. 2 Constant TES experiments. (Top) The table reports the concentration of the different sizes of AuNPs in food, according to the different dose metrics (TES, Molarity, or TNN). In particular, in these experiments, the AuNPs concentration was adjusted to obtain the same TES values among the differently sized AuNPs. (Bottom) Lifespan experiments with constant TES. The different lifespan curves are relative to six populations of Drosophila treated with food containing 5, 15, 40 and $80 \mathrm{~nm}$ of AuNPs, supernatant (SN) and control (CTRL). The inset in the graph shows the half-life ( $\tau_{50}$ ) relative to each treatment (supernatant is not reported because it has the same half-life value of control).

On the other hand, the experiments evaluating the impact of the Total Number of Nanoparticles (TNN) ingested by the flies are displayed in Fig. 3. In this case, $N$ was kept constant (i.e., constant AuNPs molar concentration), while the TES of the particles dispersed in the culture food varied among the differently sized NPs (see table in Fig. 3). This means that, in this latter case, the surface area of the particles ingested by the Drosophila was very different for the four families of AuNPs, spanning nearly 3 orders of magnitude from the $5 \mathrm{~nm}$ to the $80 \mathrm{~nm}$ particles (table in Fig. 3). Consistent with the above results, we observed that the lifespan curves of the differently treated populations were basically superimposed, despite the huge variation of the TES (Fig. 3), suggesting a direct dependence of the toxic effects on the particle concentration. This indicates that, for a given TNN, the toxicity is independent of the particles size (surface), thus further supporting the finding that $T[S(R), N]=T[N]$. The TNN selected in this case resulted in a lifespan reduction from 82 days to $c a$. $62-63$ days.

Lifespan data were supported by additional biological experiments performed on the AuNPs-treated Drosophila, namely evaluation of the reproductive performances of the flies, generation of reactive oxygen species (ROS), possible occurrence of DNA damage (by TUNEL assay), and quantitative analyses of stress markers (by real-time qPCR). In Fig. 4, we report the results obtained for $15 \mathrm{~nm}$ AuNPs (used at the same concentration, $100 \mathrm{pM}$, of the previous lifespan experiment). We found that the ingestion of AuNPs elicits a significant fertility reduction in Drosophila, both in male and female individuals (down to $c a$. $65 \%$ with respect to the control, Fig. 4A). On the other hand,
AuNPs treatment induces ROS generation (Fig. 4B) and clear DNA fragmentation in the gastrointestinal tissues (Fig. 4C). Additionally, we observed a dramatic increase of the expression level of two well known stress markers (Hsp70 and Hsp83) in treated flies (Fig. 4D). ${ }^{21,23}$ Overall, these results clearly suggest a remarkable in vivo toxicity of citrate-capped AuNPs, in line with the strong lifespan reductions discussed above. The detailed mechanisms underlying the in vivo toxicity processes require further extensive investigations. However, the available experimental data suggest a mechanism of general toxicity induced by the NPs, involving the whole organism (as revealed by the lifespan and fertility data). This assumption is supported by the realtime $\mathrm{qPCR}$ results that showed an overexpression of genes generally involved in restoring cellular homeostasis following physical, chemical and/or physiological stress. Consistently, the higher levels of ROS detected in the NPs-treated organisms also indicate a process of generalized damage occurring at the cellular level. Such NPs-induced stress is also responsible for some specific toxic effects, such as generation of DNA damage as well as presence of apoptotic events. Importantly, we found that, for a fixed TNN, the level of toxicity is the same for different sizes of AuNPs (in the 5-80 $\mathrm{nm}$ range), regardless of the different surface areas (not shown). This means that, despite that nanomaterials toxicity is mostly due to their increased surface area with respect to their macroscopic counterparts (for instance, the same mass of nanometre scale particles has a surface area that is 6 orders of magnitude larger than millimetre scale particles), in the sub$100 \mathrm{~nm}$ range the surface area does not play a primary role. Such a novel finding may have several remarkable implications for 


\begin{tabular}{|c|c|c|c|}
\hline \multicolumn{4}{|c|}{ Constant TNN } \\
\hline $\begin{array}{l}\text { AuNPs size } \\
\text { (nm) }\end{array}$ & $\begin{array}{c}\text { TES } \\
\text { Total Exposed Surface } \\
\text { area of AuNPs in Food } \\
\left(\mathrm{nm}^{2} / \mu \mathrm{l}\right)\end{array}$ & $\begin{array}{l}\text { Molarity } \\
\text { Concentration of } \\
\text { AuNPs in Food } \\
\text { (pM) }\end{array}$ & $\begin{array}{c}\text { TNN } \\
\text { Total Number of } \\
\text { AuNPs in Food } \\
\text { (AuNPs Number/ } \mu \text { l) }\end{array}$ \\
\hline 5 & $4.73 \times 10^{9}$ & 100 & $6.02 E+07$ \\
\hline 15 & $4.25 \times 10^{10}$ & 100 & $6.02 E+07$ \\
\hline 40 & $3.02 \times 10^{11}$ & 100 & $6.02 E+07$ \\
\hline 80 & $1.21 \times 10^{12}$ & 100 & $6.02 E+07$ \\
\hline
\end{tabular}

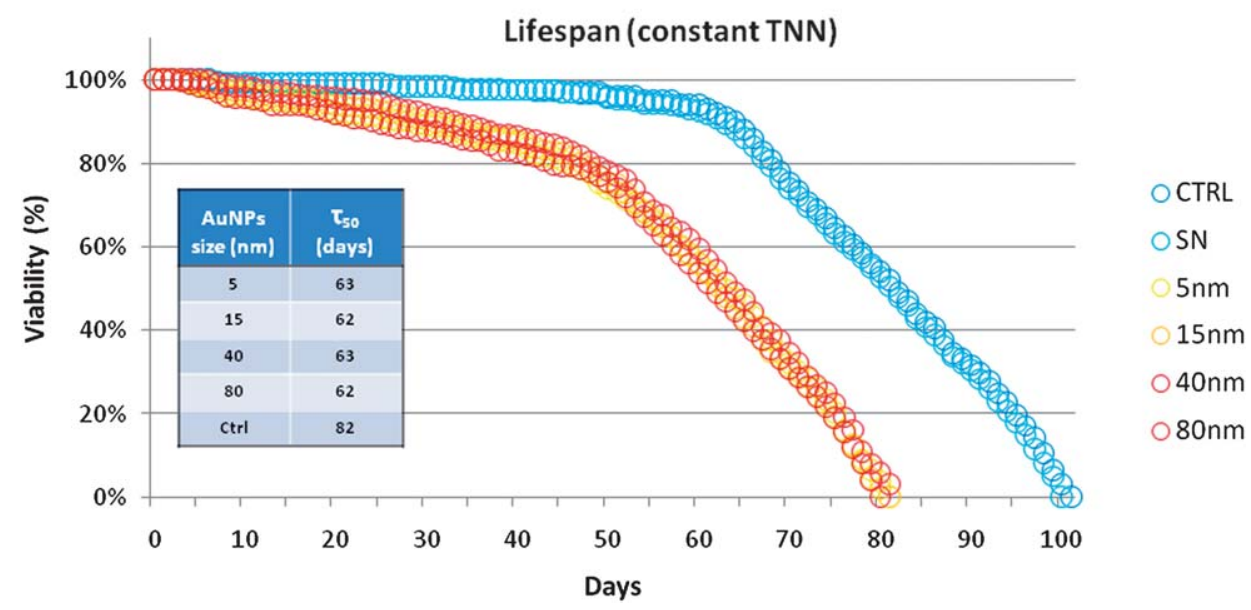

Fig. 3 Constant TNN experiments. (Top) The table reports the concentration of the different sizes of AuNPs in food, according to the different dose metrics (TES, molarity, or TNN). In particular, in these experiments, the AuNPs concentration was adjusted to obtain the same TNN values among the differently sized AuNPs. (Bottom) Lifespan experiments with constant TNN. The different lifespan curves are relative to six populations of Drosophila treated with food containing 5, 15, 40 and $80 \mathrm{~nm}$ of AuNPs, supernatant (SN) and control (CTRL). The inset in the graph shows the half-life ( $\tau_{50}$ ) relative to each treatment (supernatant is not reported because it has the same half-life value of control).

a wide range of nanotoxicology/nanomedicine applications. Although this concept partly disagrees with the commonly accepted paradigm of surface area in nanotoxicology, it is consistent with many recent size-dependent toxicity studies performed by several groups on different nanomaterials, in which the NPs toxicity was not found to scale with their surface area. ${ }^{24-27}$

Once we defined the main factor that induces the variation of the toxic effects (establishing the dependence of the toxicity functional $T$ on the $N$ parameter alone), we tried to physically assess and quantify the toxic effects of our AuNPs observed in vivo, determining a toxicity factor of the nanoparticles. To this aim, we performed dose dependence toxicity experiments on our $15 \mathrm{~nm}$ AuNPs (there are no differences in the 5-80 nm size range), defining their specific toxicity parameters. Experimental results are reported in Fig. 5 (in the concentration range from $5 \mathrm{pM}$ to $2 \mathrm{nM}$ ), in terms of the reduction of the average lifespan $\left(\tau_{50}\right)$. The adverse effects of AuNPs are rather high in the low concentration range (up to $20 \mathrm{pM}$ ), while a plateau of $c a$. $55 \%$ (representing the maximum fractional lifespan reduction) is evident at higher AuNPs doses, suggesting a two-component behavior, with a fast toxic response followed by a slow viability decrease. Experimental data were, in fact, successfully fitted $\left(R^{2}=0.999\right)$ by a bi-exponential decay curve (Fig. 5), clearly indicating the occurrence of remarkable toxic mechanisms at low concentration, represented by the second component $\left(k_{2}=0.21\right)$, accompanied by a much slower decay kinetics, $c a .2$ orders of magnitude $\left(k_{1}=4 \times 10^{-3}\right)$, which accounts for a nearly constant behavior for AuNPs concentrations higher than $500 \mathrm{pM}$. This dose dependent response to the citrate-capped AuNPs allowed us to define the toxicity parameters of such specific NPs. To this purpose, we associated the toxicity factor of such nanomaterial to the slope of the viability decay curve at low concentration, namely $y_{x \rightarrow 0}^{\prime}(x)$. Under the reasonable approximation $k_{2} \gg k_{1}$, we calculated that

$$
\begin{gathered}
y^{\prime}(0)=-k_{2}\left(100+A_{1}-y_{0}\right)(\text { for a normalized curve }, \\
\left.A_{2}=100-A_{1}-y_{0}\right) .
\end{gathered}
$$

Hence, the specific toxicity of a nanomaterial can be described by the following three parameters:

$$
\left\{\begin{array}{l}
k_{2} \text { (fast component) } \\
y_{0} \text { (plateau, i.e., resistance to high NPs doses) } \\
A_{1} \text { (coefficient of the slow component) }
\end{array}\right.
$$

$k_{2}$ accounts for the rapid decrease of the viability in the first part of the curve, while its contribution rapidly approaches zero as the concentration increases (in this case, the fast component is nearly zero above $20 \mathrm{pM}$ concentration). $y_{0}$ indicates the maximum lifespan reduction that Drosophila may undergo upon ingestion of high doses of NPs (thus representing a sort of resistance to the toxicity), while $A_{1}$ is important to determine the behavior of the 

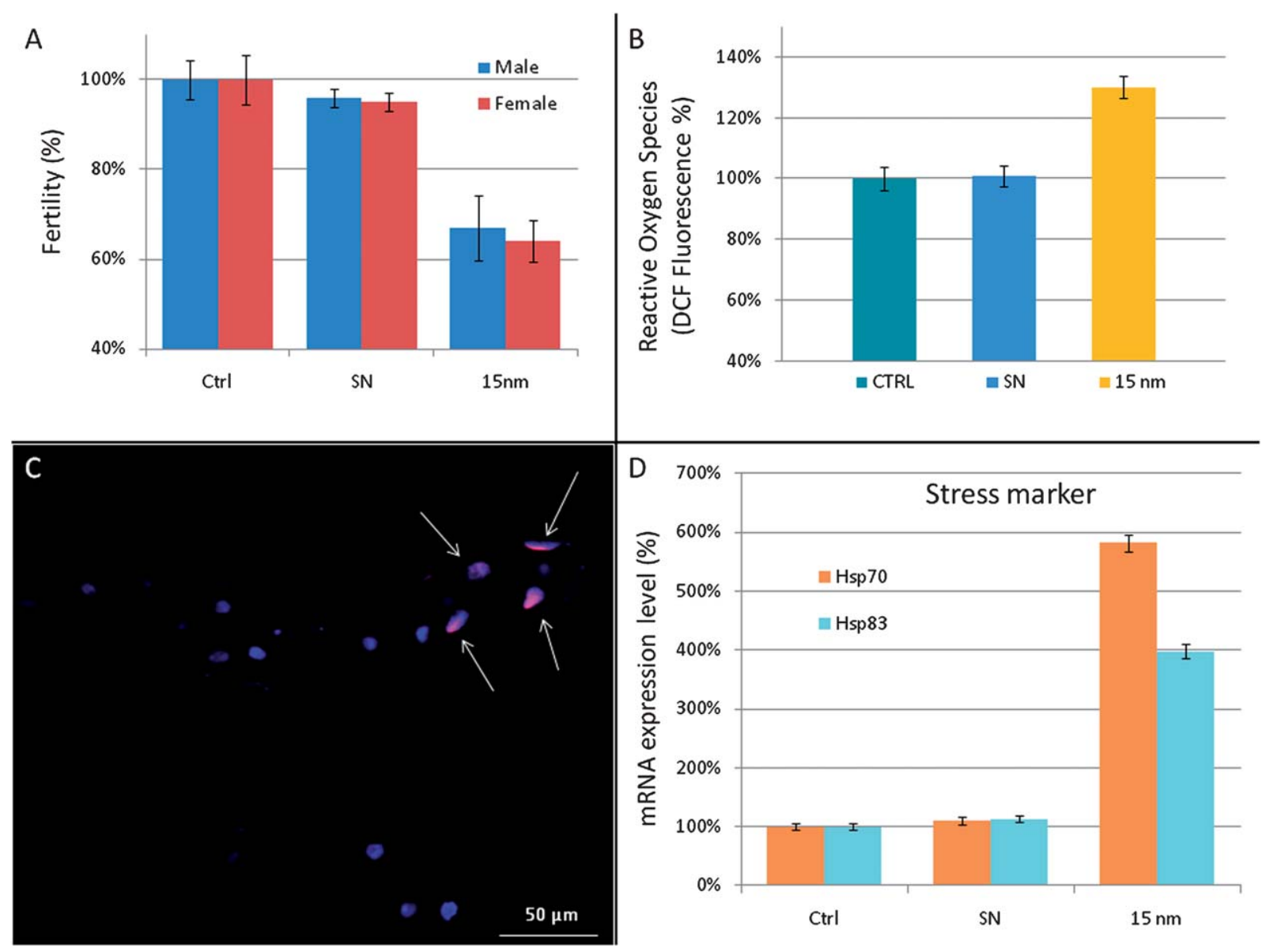

Fig. 4 Representative biological stress effects observed in Drosophila organism upon ingestion of $15 \mathrm{~nm}$ AuNPs (100 pM). (A) Fertility test: male and female treated flies show a significant reduction in the reproductive performance (down to $c a .65 \%$ with respect with the control), while $\mathrm{SN}$ treated flies show a fertility similar to the control. (B) Reactive Oxygen Species (ROS) measurements: $15 \mathrm{~nm}$ AuNPs treated flies show an increased generation of ROS ( $c$ a. 30\% higher than the control), while SN treated flies did not show any differences in ROS generation. (C) TUNEL assay: representative confocal image of Drosophila gastrointestinal tissues. White arrows indicate the TUNEL-positive nuclei colored in red, while normal nuclei are colored in blue. (D) Real-Time qPCR experiments: mRNA expression level of two stress marker proteins (Hsp70 and Hsp83) results over-expressed in AuNPs treated flies (ca. $600 \%$ and $400 \%$, respectively, compared to the control). In SN treated flies, the mRNA expression level resulted not altered and comparable with the control.

slow high dose region. Both $y_{0}$ and $A_{1}$ have also an important role in determining the initial slope of the toxicity decay curve.

Therefore, a nanomaterial will be highly toxic when the following set of toxicity parameters is found:

$$
\text { HIGH TOXICITY } \longrightarrow\left\{\begin{array}{lll}
k_{2} & \text { High } & (\text { e.g., }>0.25) \\
y_{0} & \text { Small } & (\text { e.g., }<40) \\
A_{1} & \text { High } & (\text { e.g., }>30)
\end{array}\right.
$$

In this case, the toxicity factor $y^{\prime}(0)$ will be very negative, and the resistance value will be very low. For instance, a very toxic nanomaterial could be characterized by a toxicity factor of ca. $-45\left(k_{2}=0.40 ; y_{0}=25 ; A_{1}=40\right)$, while for our citrate-capped AuNPs $y^{\prime}(0)$ is $c a$. -15 .

Using our AuNPs as a reference toxicity standard, we can define different regions in the multiparametric space of toxicity, and thus different toxicity levels for other nanomaterials to be tested (Fig. 6). This is exemplified by the three main regions in
Fig. 6, ranging from highly toxic to biocompatible nanomaterials (the latter producing no or minor effects in terms of reduced viability, i.e. less than $5 \%$ with respect to the control). Such a model was exploited to test and classify two different nanomaterials, namely CdSe/ZnS core/shell carboxyl-terminated QDs and the same AuNPs with PEG functionalization. As reported in Fig. 6, QDs exhibited a strong lifespan reduction ( $\mathrm{ca}$. 35\% less than the control, at a concentration as low as $85 \mathrm{pM}$ ), falling in the dark red region of the toxicity space, and being classified as highly toxic nanomaterials. On the other hand, the pegylation procedure of the AuNPs was found to make them much more inert than the citrate-capped counterparts, with a negligible lifespan reduction $(\sim 3 \%)$ at $150 \mathrm{pM}$ concentration, making them eligible as biocompatible materials. More in general, an accurate dose dependence study of any nanomaterial would lead also to a quantitative determination of its toxicity potential by means of the calculation of its toxicity factor.

In conclusion, we have demonstrated that in the $5-80 \mathrm{~nm}$ size range the surface area of the NPs does not play a primary role in determining the particle toxicity and, thus, the dose metrics; on 


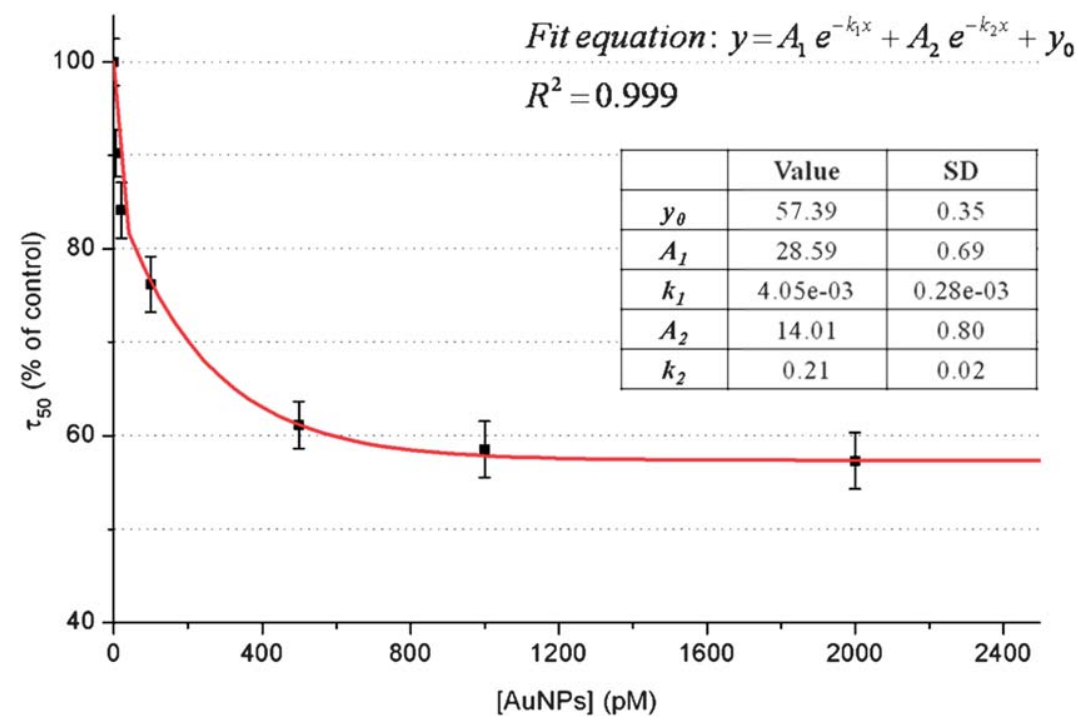

Fig. 5 Dose dependent toxicity response of $15 \mathrm{~nm}$ AuNPs, expressed as fractional reduction of the fly lifespan as compared to the control. The red line represents a bi-exponential fitting of the experimental data. The equation and the fitting parameters are reported in the figure (top right and in the table).

the other side, the number of NPs seems to be the crucial parameter in inducing toxicity. Based on these findings, a physical quantification of the NPs toxicity has been developed through the toxicity factor, possibly opening the way to a systematic classification of engineered and ambient nanomaterials with similar characteristics. This, in turn, may lead to interesting developments in risk assessment and regulatory approval, as well as in a wide range of nanomedicine applications. However, in the case of different nanomaterials, in which other characteristics, such as extensive aggregation, shape factors, or strong release of ions, play a key role (like, for instance, CNTs or AgNPs) our model may present specific limitations. In this respect, further investigations are needed in order to develop more advanced models and toxicity factors suitable also for polydispersed, aggregated or non-spherical nanomaterials.

\section{Methods}

\section{AuNPs synthesis}

All glassware and the magnetic stir-bar were thoroughly washed with aqua regia $\left(\mathrm{HCl}\right.$ and $\mathrm{HNO}_{3}$ in a $3: 1$ volumetric ratio). Colloidal $5 \mathrm{~nm}$ citrate-capped AuNPs were prepared in a round bottom flask with $100 \mathrm{~mL}$ ice-cold aqueous solution containing $0.25 \mathrm{mM} \mathrm{HAuCl}{ }_{4}$ (Sigma-Aldrich) and $0.25 \mathrm{mM}$ trisodium citrate (Sigma-Aldrich). Next, $0.6 \mathrm{~mL}$ of ice-cold, freshly

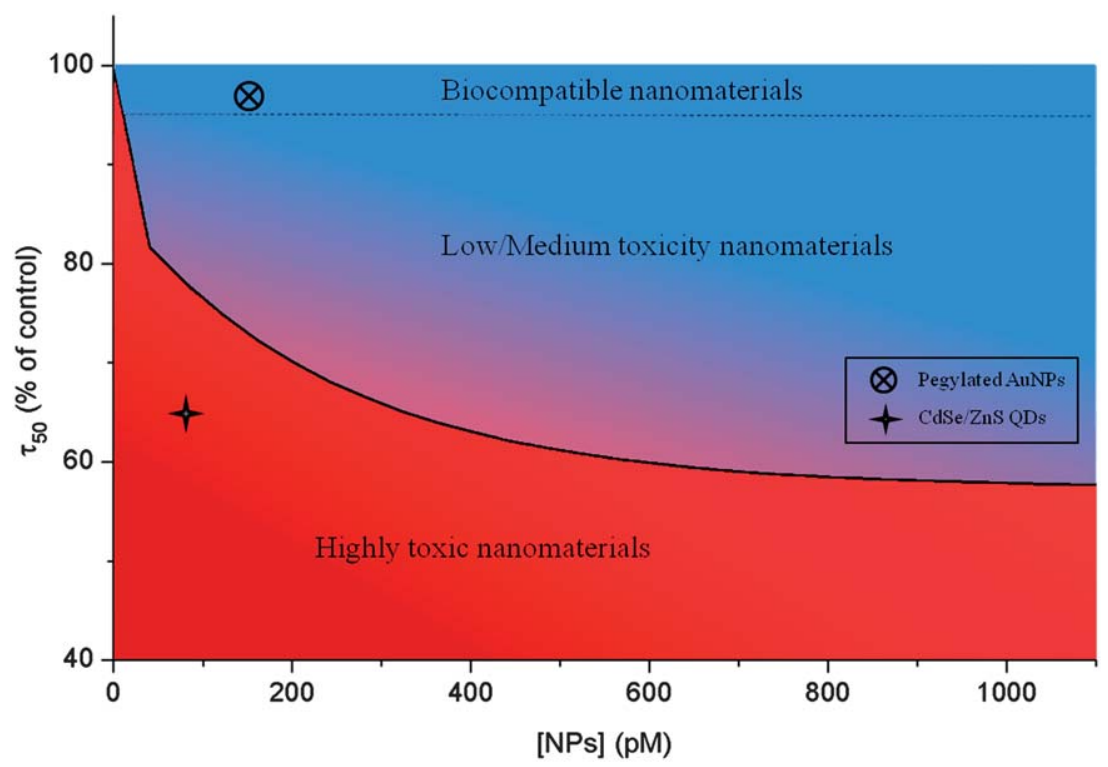

Fig. 6 Schematic picture representing the different toxicity regions of the different nanomaterials (highly toxic, low/medium toxic, biocompatible). The black line represents the reference toxicity curve (bi-exponential fit) of the $15 \mathrm{~nm}$ citrate-capped AuNPs. The toxicity levels of pegylated AuNPs (150 pM) and carboxyl terminated QDs (85 pM) are also shown. 
prepared 0.1 $\mathrm{M} \mathrm{NaBH}_{4}$ (Sigma-Aldrich) solution was added to the solution while stirring. The solution turned red-brown immediately after the addition of the reducing agent, indicating nanoparticles formation. Here, citrate serves only as a capping agent since it cannot reduce the gold salt at this temperature $\left(4{ }^{\circ} \mathrm{C}\right)$. Colloidal $15 \mathrm{~nm}$ citrate-capped AuNPs were synthesized by the classical Turkevich-Frens method, ${ }^{28,29}$ using sodium citrate as the reducing agent. Briefly, $150 \mathrm{~mL}$ of $0.25 \mathrm{mM}$ aqueous solution of $\mathrm{HAuCl}_{4}$ was heated to boil while stirring. Then, $2.8 \mathrm{~mL}$ of $1 \%$ aqueous solution of sodium citrate were added. The solution was kept gently boiling till a red wine color appeared. AuNPs of $40 \mathrm{~nm}$ and $80 \mathrm{~nm}$ were prepared according to a two-step seed-mediated $\operatorname{method}^{30}$ which allows the enlargement of $15 \mathrm{~nm}$ AuNPs (seeds) thanks to the property of $\mathrm{NH}_{2} \mathrm{OH}$ to efficiently reduce $\mathrm{Au}^{3+}$ to bulk metal in the presence of Au surface. ${ }^{31}$ The synthesis was performed by adding $2 \mathrm{~mL}$ of aqueous $40 \mathrm{mM}$ hydroxylamine sulfate (Sigma-Aldrich) and different numbers of $15 \mathrm{~nm}$ AuNPs (seeds) into $200 \mathrm{~mL}$ aqueous solution. The solution was kept under vigorous stirring and then $25 \mathrm{~mL}$ of $2 \mathrm{mM}$ aqueous solution of $\mathrm{HAuCl}_{4}$ was dropwise added to seeds solution $\left(1 \mathrm{~mL} \mathrm{~min}{ }^{-1}\right)$. After the addition of $\mathrm{HAuCl}_{4}$ solution was finished, stirring was continued for $30 \mathrm{~min}$, and then $12 \mathrm{~mL}$ of $1 \%$ aqueous solution of trisodium citrate was injected to stabilize AuNPs by the weak capping effect of such chemical. To minimize the presence of solvent and unreacted reagents, all the solutions were immediately centrifuged for $15 \mathrm{~min}$, then 5,15 , 40 and $80 \mathrm{~nm}$ AuNPs were suspended in ultrapure, sterile water. Before their use, NPs were filtered using $0.22 \mu \mathrm{m}$ syringe filters (Fluoropore PTFE membrane, purchased form Millipore Corp.) under a laminar flow biological safety cabinet, to ensure sterility.

\section{AuNPs characterization}

AuNPs size and shape were assessed by TEM analyses. The 300 mesh carbon coated copper grid was cast with a few drops of citrate-capped AuNPs and vacuum dried. TEM images of each sample were collected using a JEOL 1011 transmission electron microscope with an accelerating voltage of $100 \mathrm{kV}$.

Surface Plasmon Resonance (SPR) spectra were recorded using a Cary 300 Bio double-beam spectrophotometer. The AuNPs concentrations were obtained by using the intensity of maximum absorption of the SPR peak and molar extinction coefficients reported in literature. ${ }^{32,33}$ Further characterizations were performed by Dynamic Light Scattering (DLS) and $\zeta$ potential analyses using a Zetasizer Nano-ZS instrument (Malvern Instruments) equipped with a $4.0 \mathrm{mV}-\mathrm{He}-\mathrm{Ne} 633 \mathrm{~nm}$ laser.

\section{Drosophila melanogaster strain and culture conditions}

The flies and larvae of wild-type Drosophila melanogaster (Oregon $\mathrm{R}+$ ) were cultured at $24 \pm 1{ }^{\circ} \mathrm{C}$ on standard Drosophila food, containing agar, corn meal, sugar, yeast and nepagin (methyl- $p$-hydroxybenzoate).

\section{Gold nanoparticles exposure}

AuNPs were formulated in the diet. Four different sizes (5, 15, 40 and $80 \mathrm{~nm}$ ) of AuNPs were dispersed in the food and used for experiments. In particular, the solution containing AuNPs was added to the food before solidification, mixed strongly and finally poured into vials. TEM analyses showed that the AuNPs in the Drosophila food maintained a good degree of monodispersity (data not show). For the lifespan of AuNPs treated flies, we carried out two types of approaches: (i) Total Number of Nanoparticles (TNN), in which we used the same concentration $\left(6.02 \times 10^{7}\right.$ particles per $\left.\mu \mathrm{L}\right)$ of different sizes of AuNPs, and (ii) Total Exposed Surface (TES) area, in which we used different concentrations (5 nm: 900 pM; $15 \mathrm{~nm}$ : 100 pM; $40 \mathrm{~nm}: 14$ pM; $80 \mathrm{~nm}: 3.5 \mathrm{pM})$ but the same total exposed surface area $(4.25 \times$ $\left.10^{10} \mathrm{~nm}^{2} \mu \mathrm{L}^{-1}\right)$. With the same modality, we prepared food with AuNPs supernatant (SN). SN was obtained by centrifugation of the NPs solution at $13400 \mathrm{rpm}$ for $30 \mathrm{~min}$. This preparation was used to exclude the presence of toxic compounds in the solution containing AuNPs.

Assuming that Drosophila typically eats $1.5 \mu \mathrm{L}$ per day of food, ${ }^{24}$ the dose of $15 \mathrm{~nm}$ AuNPs (100 pM) administered to the flies was $3.08 \mu \mathrm{g} \mathrm{g}^{-1}$ per day. The other doses used in the experiments can be calculated from the above value (in any case, the range of the dose varied from $0.154 \mu \mathrm{g} \mathrm{g}^{-1}$ to $467 \mu \mathrm{g} \mathrm{g}^{-1}$ per day).

\section{Lifespan experiments}

For longevity analyses, newly eclosed flies were collected and housed at a density of 20 males and 20 females, separately, per vial. At least 10 vials were used per each treatment (total of 100 male and 100 female flies per lifespan) for a total number of 1200 flies in the TNN experiment and 1200 in the TES experiment. Flies were counted daily and transferred every 4 days in new vials containing fresh food. These experiments were performed using normal food, treated food containing AuNPs supernatant (SN), and treated food containing AuNPs of different size.

\section{Fertility and reproductive performance}

The method of Gayathri and Krishnamurthy ${ }^{34}$ was followed with some modifications. Virgin flies emerging from the control, SN and AuNPs treated food were isolated and pair mated in normal food vials. Pair mating was performed in two different conditions per treatment group (30 pairs of flies were taken per each treatment group): (1) treated males with normal females; and (2) normal males with treated females. Flies were transferred into fresh vials every day for the subsequent ten days. The total number of flies eclosed from the eggs laid during these ten days of pair mating was counted. The mean number of flies emerged per pair for ten days gave a measure of the reproductive performance.

\section{Measurement of ROS}

Molecular oxygen is the key to aerobic life but it may be also converted into cytotoxic byproducts, usually referred to as reactive oxygen species (ROS), that have been reported to play a major role in the toxicity of several xenobiotics, including metals and pesticides. ${ }^{35}$ Then, in our study, the measurement of ROS represents an important tool to assess the cellular toxicity induced by the AuNPs in Drosophila organism. Intracellular ROS were measured using a dye, 2,7-dichlorofluorescein diacetate (DCFH-DA; Sigma, St Louis, MO, USA). 20 five-day-old 
flies were homogenized in tubes containing $1 \mathrm{~mL}$ PBST (PBS containing $0.1 \%$ Tween-20). The supernatant of each sample was divided in two different vials: the first vial was transferred into a 96-well plate. After adding $50 \mu \mathrm{M}$ DCF-DA to the samples, the plate was read every $5 \mathrm{~min}$ for $15 \mathrm{~min}$ by a fluorescent microplate reader (FLUOstar Optima, BMG Laboratory, Offenberg, Germany) for the quantification of the fluorescence (excitation $485 \mathrm{~nm}$; emission $520 \mathrm{~nm}$ ). The second vial was used for quantification of the protein crude extract. Following centrifugation at $2300 \mathrm{~g}$ for $15 \mathrm{~min}$ at $4{ }^{\circ} \mathrm{C}$ in the presence of a protease inhibitor, the supernatant was quantified by Bradford method. ${ }^{36}$ The amount of proteins in the crude extraction was used to normalize the relative DCFH fluorescence measured in each sample. Three independent experiments with 20 flies in each experiment were performed.

\section{TUNEL assay}

Midgut was dissected in Ringer's Buffer and fixed in 3.7\% paraformaldehyde in PBS for 3 hours. After washing in PBT (PBS, 0.1\% Tween 20), midgut was heated in $500 \mu \mathrm{l} \mathrm{PBT}$ for 5 min to approximately $85{ }^{\circ} \mathrm{C}$ to enhance the efficiency of the labeling reaction. Midgut was then incubated in PBT containing $50 \mathrm{mM} \mathrm{NH}_{4} \mathrm{Cl}$ for $10 \mathrm{~min}$, and permeabilized in PBS $0.3 \%$ Triton X 100 overnight. Midgut was processed by Click-iT TUNEL Alexa Fluor647 Imaging Assay (Invitrogen), according to the manufacturer's instructions. Midgut was equilibrated for $10 \mathrm{~min}$ in TdT TUNEL buffer and then added to TdT reaction cocktail, containing TdT enzyme and a modified dUTP, and incubated for $60 \mathrm{~min}$ at $37^{\circ} \mathrm{C}$. After incubation, midgut was washed twice with $3 \%$ BSA in PBS for 2 minutes each. Subsequently, sample was incubated with Click-iT reaction cocktail and incubated for $30 \mathrm{~min}$ at room temperature, protected from light. Then, the Click-iT reaction cocktail was removed and tissue was washed with 3\% BSA in PBS for 5 minutes and added $1 \times$ Hoechst 33342 solution for $15 \mathrm{~min}$ at room temperature. These samples were then characterized by confocal microscopy (Leica TCS-SP5 AOBS).

\section{Quantitative real-time PCR}

Third instar larvae extracts were prepared by homogenizing larvae in groups of 10 in cold solution of RNAlater (Sigma). Total RNA was isolated from flies using Tri-reagent (Sigma), the amount of RNA in each sample was determined by absorbance at $260 \mathrm{~nm}$ (NanoDrop 2000c, Thermo scientific), and RNA quality was analyzed using agarose gel electrophoresis (1.2\%). First-strand cDNA was prepared from $3 \mu \mathrm{g}$ total RNA using Enhanced Avian Reverse Transcriptase (Sigma Aldrich) and oligo $(\mathrm{dT})_{18}$ primers in $20 \mu \mathrm{l}$ reaction volume. Real-time quantitative PCR was performed with an ABI 7500 thermal cycler (Applied Biosystem), using SYBR Green-based detection. Melting curves were examined after PCR runs to confirm the specific amplification. For each gene we used $10 \mathrm{ng}$ of cDNA mixed with $10 \mu \mathrm{L}$ of $10 \times$ Express SYBR Green qPCR SuperMix premixed with ROX (Invitrogen), $2 \mu \mathrm{L}$ of $4 \mu \mathrm{M}$ gene specific primers mix and $7 \mu \mathrm{L}$ of DEPC-treated water. Reaction conditions for all genes were: initial denaturation at $95^{\circ} \mathrm{C}$ for $10 \mathrm{~min}$, followed by 40 cycles of $15 \mathrm{~s}$ at $95^{\circ} \mathrm{C}, 1 \mathrm{~min}$ at $60^{\circ} \mathrm{C}$. This program was followed by a melting curve program $\left(60-95{ }^{\circ} \mathrm{C}\right.$ with a heating rate of $0.1{ }^{\circ} \mathrm{C} \mathrm{s}^{-1}$ and continuous fluorescence measurements). Relative expression was calculated from cycle threshold values ( $\Delta \Delta \mathrm{Ct}$ method) using RpL32 ribosomal RNA expression as the internal control for each sample. The primers used in the Real-Time qPCR analyses were designed by online Primer-BLAST software of NCBI (http://www.ncbi.nlm.nih.gov/ tools/primer-blast/). In particular, we used Rpl32 primer (forward: CGAGTTGAACTGCCTTCAAGATGACCA; reverse: CCGACTGGTGGCGGATGAAGTG) that amplify the target sequence NM_001144656.1; hsp70 primer (forward: AGGGTCAGATCCACGACATC; reverse: CGTCTGGGT TGATGGATAGG) that amplify the target sequences NM_176486.1; NM_169469.1; NM_169441.1; NM_141952.1; NM_080188.2; NM_080059.2 and hsp83 primer (forward: TGGAGGCTCTGCAGGCTGGT; reverse: GGCGAC CAGGTAGGCGGAGT) specific for the target sequence NM_079175.2. All the target sequences are reported as NCBI accession number.

\section{Acknowledgements}

The authors gratefully acknowledge M. A. Malvindi, L. Rizzello, B. Sorce, S. Shiv Shankar, L. Martiradonna, and R. Mastria for useful discussions and help during experiments, and V. Fiorelli for the expert technical assistance.

\section{References}

1 G. Oberdorster, J. Intern. Med., 2010, 267, 89-105.

2 G. Maiorano, S. Sabella, B. Sorce, V. Brunetti, M. A. Malvindi, R. Cingolani and P. P. Pompa, ACS Nano, 2010, 4, 7481-7491.

3 T. Cedervall, I. Lynch, S. Lindman, T. Berggard, E. Thulin, H. Nilsson, K. A. Dawson and S. Linse, Proc. Natl. Acad. Sci. U. S. A., 2007, 104, 2050-2055.

4 M. Lundqvist, J. Stigler, G. Elia, I. Lynch, T. Cedervall and K. A. Dawson, Proc. Natl. Acad. Sci. U. S. A., 2008, 105, 1426514270.

5 S. Lanone, F. Rogerieux, J. Geys, A. Dupont, E. Maillot-Marechal, J. Boczkowski, G. Lacroix and P. Hoet, Part. Fibre Toxicol., 2009, 6, DOI: $10.1186 / 1743-8977-6-14$

6 P. P. Pompa, G. Vecchio, A. Galeone, V. Brunetti, S. Sabella, G. Maiorano, A. Falqui, G. Bertoni and R. Cingolani, Nano Res., 2011, DOI: $10.1007 / \mathrm{s} 12274-011-0095-\mathrm{z}$.

7 D. Bhargav, M. Pratap Singh, R. C. Murthy, N. Mathur, D. Misra, D. K. Saxena and D. Kar Chowdhuri, Ecotoxicol. Environ. Saf., 2008, 69, 233-245.

8 M. D. Rand, Neurotoxicol. Teratol., 2010, 32, 74-83.

9 M. Ahamed, R. Posgai, T. J. Gorey, M. Nielsen, S. M. Hussain and J. J. Rowe, Toxicol. Appl. Pharmacol., 2010, 242, 263-269.

10 E. Demir, G. Vales, B. Kaya, A. Creus and R. Marcos, Nanotoxicology, 2011, DOI: 10.3109/17435390.2010.529176.

11 X. Liu, D. Vinson, D. Abt, R. H. Hurt and D. M. Rand, Environ. Sci. Technol., 2009, 43, 6357-6363.

12 F. Hirth, CNS Neurol. Disord.: Drug Targets, 2010, 9, 504-523.

13 A. M. Brumby and H. E. Richardson, Nat. Rev. Cancer, 2005, 5, 626639.

14 M. Vidal and R. L. Cagan, Curr. Opin. Genet. Dev., 2006, 16, 10-16.

15 K. Ocorr, T. Akasaka and R. Bodmer, Mech. Ageing Dev., 2007, 128, $112-116$.

16 A. T. Haselton and Y. W. C. Fridell, Aging, 2010, 2, 523-526.

17 A. M. Alarco, A. Marcil, J. Chen, B. Suter, D. Thomas and M. Whiteway, J. Immunol., 2004, 172, 5622-5628.

18 V. Leclerc and J. M. Reichhart, Immunol. Rev., 2004, 198, 59-71.

19 L. T. Reiter, L. Potocki, S. Chien, M. Gribskov and E. Bier, Genome Res., 2001, 11, 1114-1125.

20 K. A. Matthews, T. C. Kaufman and W. M. Gelbart, Nat. Rev. Genet., 2005, 6, 179-193. 
21 M. P. Singh, M. M. Reddy, N. Mathur, D. K. Saxena and D. K. Chowdhuri, Toxicol. Appl. Pharmacol., 2009, 235, 226-243.

22 W. W. Ja, G. B. Carvalho, E. M. Mak, N. N. de la Rosa, A. Y. Fang, J. C. Liong, T. Brummel and S. Benzer, Proc. Natl. Acad. Sci. U. S. A., 2007, 104, 8253-8256.

23 S. C. Gupta, A. Sharma, M. Mishra, R. K. Mishra and D. K. Chowdhuri, Life Sci., 2010, 86, 377-384.

24 Y. Pan, S. Neuss, A. Leifert, M. Fischler, F. Wen, U. Simon, G. Schmid, W. Brandau and W. Jahnen-Dechent, Small, 2007, 3, 1941-1949.

25 Y. Y. Ye, J. W. Liu, M. C. Chen, L. J. Sun and M. B. Lan, Environ Toxicol. Pharmacol., 2010, 29, 131-137.

26 X. Yang, J. Liu, H. He, L. Zhou, C. Gong, X. Wang, L. Yang, J. Yuan, H. Huang, L. He, B. Zhang and Z. Zhuang, Part. Fibre Toxicol., 2010, 7, 1 .

27 D. B. Warheit, T. R. Webb, C. M. Sayes, V. L. Colvin and K. L. Reed, Toxicol. Sci., 2006, 91, 227-236.
28 J. Turkevich, P. C. Stevenson and J. Hillier, Discuss. Faraday Soc., 1951, 11, 55-75.

29 G. Frens, Nat. Phys. Sci., 1973, 241, 20-22.

30 X. Q. Zou, E. B. Ying and S. J. Dong, Nanotechnology, 2006, 17, $4758-4764$.

31 G. Stremsdoerfer, H. Perrot, J. R. Martin and P. Clechet, J. Electrochem. Soc., 1988, 135, 2881-2886.

32 J. S. Lee, S. I. Stoeva and C. A. Mirkin, J. Am. Chem. Soc., 2006, 128, 8899-8903.

33 X. O. Liu, M. Atwater, J. H. Wang and Q. Huo, Colloids Surf., B, $2007, \mathbf{5 8}, 3-7$.

34 M. V. Gayathri and N. B. Krishnamurthy, Environ. Res., 1981, 24, 89-95.

35 J. J. Fortunato, G. Feier, A. M. Vitali, F. C. Petronilho, F. Dal-Pizzol and J. Quevedo, Neurochem. Res., 2006, 31, 671678.

36 M. M. Bradford, Anal. Biochem., 1976, 72, 248-254. 